\title{
Mapping Alteration Caused by Hydrocarbon Microseepages in Patrick Draw area Southwest Wyoming Using Image Spectroscopy and Hyperspectral Remote Sensing
}

Grant/Cooperative Agreement No. DE-FG26-05NT42494

\author{
PI: Shuhab D. Khan
}

Final Report

(Reporting period July 1, 2005 to June 30, 2006)

October 2006

Prepared by

Shuhab D. Khan, Department of Geosciences, University of Houston, 312 S\& R1, 4800 Calhoun RD. Houston, TX. 77204 


\section{DISCLAIMER}

"This report was prepared as an account of work sponsored by an agency of the United States Government. Neither the United States Government nor any agency thereof, nor any their employees, makes any warranty, express or implied, or assumes any legal liability or responsibility for he accuracy, completeness or usefulness of any information, apparatus, products, or process disclosed, or represents that its use would not infringe privately owned rights, Reference herein to any specific commercial product, process, or service by trade name, trademark, manufacturer, or otherwise does not necessarily constitute or imply its endorsement, recommendation, or favoring by the United States Government or any agency thereof. The views and opinions of authors expressed herein do not necessarily state or reflect those of the United States Government of any agency thereof" 


\begin{abstract}
Detection of underlying reservoir accumulations using remote sensing techniques had its inception with the identification of macroseeps. However, today we find ourselves relying on the detection of more subtle characteristics associated with petroleum reservoirs, such as microseeps. Microseepages are the result of vertical movement of light hydrocarbons from the reservoir to the surface through networks of fractures, faults, and bedding planes that provide permeable routes within the overlying rock. Microseepages express themselves at the surface in an array of alterations and anomalies, such as chemical or mineralogical changes in overlying soils and sediments.

Using NASA's Hyperion hyperspectral imaging sensors, this project has developed spectral and geochemical ground truthing techniques to identify and map alterations caused by hydrocarbon microseepages and to determine their relationships to the underlying geology in the Patrick Draw area of Southwest Wyoming. Training the classification of satellite imagery with spectral inputs of samples collected over previously defined areas of hydrocarbon microseepage resulted in the successful identification of an anomalous zone. Geochemical characteristics of samples that defined this anomalous zone were then compared to the remaining non-anomalous samples using $\mathrm{XRD}, \mathrm{ICP}$, spectroscopy and carbon isotope techniques.
\end{abstract}




\section{TABLE OF CONTENTS}

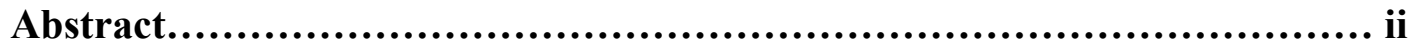

Disclaimer....................................................................... iii

1. EXECUTIVE SUMMARY .............................................. 1

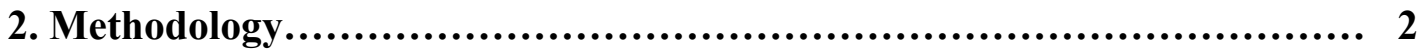

2.1. Field Work ............................................................ 2

2.2. Remote Sensing Data Analysis......................................... 2

2.2 .1 Hyperion image processing......................................... 3

2.2.2 Image Classification................................................. 3

2.3. Geochemistry.......................................................... 3

2.3.1 Laboratory Spectroscopy .......................................... 3

2.3.2. XRD Analysis......................................................... 4

2.3.3. ICP Analysis............................................................ 4

2.3.4. Carbon Isotope Analysis........................................ 4

3. RESULTS AND INTERPRETATION..................................... 4

3.1. Remote Sensing Results and Interpretation............................... 4

3.2. Geochemistry Results and Interpretation............................. 6

3.2.1 Lab Spectroscopy Results and Interpretation......................... 6

3.2.2 XRD Results and Interpretation....................................... 7

3.2.3 ICP Results and Interpretation...................................... 7

3.2.4 Carbon Isotope Results and Interpretation............................ 7

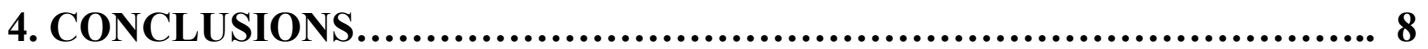

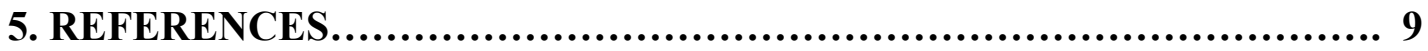

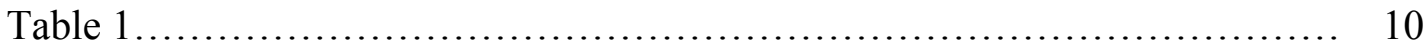

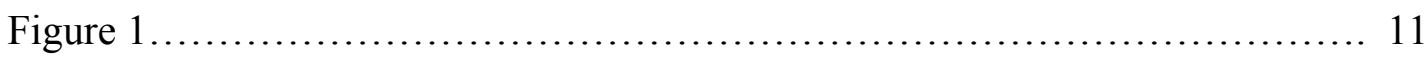

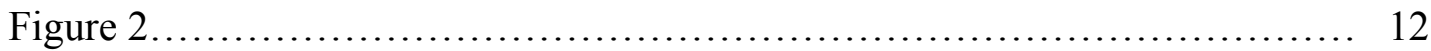

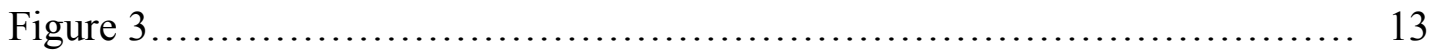

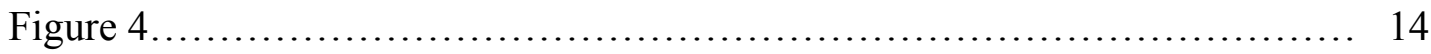

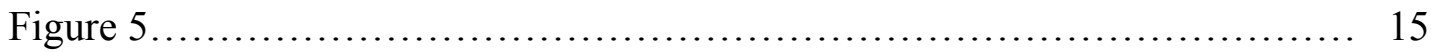

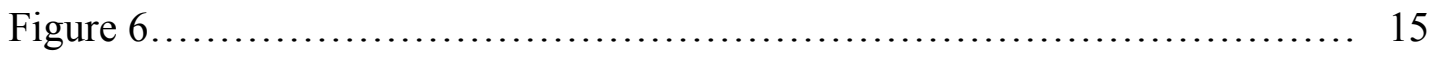




\section{EXECUTIVE SUMMARY}

Detection of underlying reservoir accumulations using remote sensing techniques had its inception with the identification of macroseeps. However, today we find ourselves relying on the detection of more subtle characteristics associated with petroleum reservoirs, such as microseeps. Microseepages are the result of vertical movement of light hydrocarbons from the reservoir to the surface through networks of fractures, faults, and bedding planes that provide permeable routes within the overlying rock. Microseepages express themselves at the surface in an array of alterations and anomalies, such as chemical or mineralogical changes in overlying soils and sediments. This project has developed remote sensing methodologies using NASA's Hyperion hyperspectral imaging sensors and ground truthing techniques in order to identify and map alterations caused by hydrocarbon microseepages whose presence has been confirmed through the use of soil gas surveys in previous studies. Also, using geochemical data, potential relationships between identified alterations and microseeping hydrocarbons was established in the Patrick Draw area so as to support connections drawn between microseepages and overlying alterations.

Training the classification of satellite imagery with spectral inputs of samples collected over previously defined areas of hydrocarbon microseepage resulted in the successful identification of an anomalous zone. Spectral Angle Mapper (SAM) as well as Mixture Tuned Matched Filter (MTMF) techniques were utilized for classification of images. Spectral Angle Mapper is a method for directly comparing image spectra to known spectral endmembers input by the user and/or defined from the image spectra itself. MTMF is a classification method that also provides a means of detecting specific materials based on matches to user endmember input or image-derived endmember spectra. However, unlike SAM, which picks the best match to a given spectrum in the image, MTMF first maximizes the response of the known endmembers and suppresses the response of the unknown background signal in the image prior to matching. SAM analysis proved to be more effective in the classification of anomalous areas, or areas that are distinct from their surroundings.

Geochemical characteristics of samples that defined this anomalous zone were then compared to the remaining non-anomalous samples using XRD, ICP, spectroscopy and carbon isotope techniques. Spectroscopy results demonstrated higher proportions of clays within the anomalous samples compared to non-anomalous samples, along with greater amounts of quartz in non-anomalous samples relative to anomalous samples. XRD analyses demonstrated the increased presence of feldspars in non-anomalous samples compared to anomalous samples. geochemical results provided evidence for the removal of soluble constituents from the weathering sites of anomalous samples versus non-anomalous samples. The $\delta^{13} \mathrm{C}$ values range from $-2.88 \%$ to as low as $-45.32 \%$

Although this project has laid the groundwork for the identification of hydrocarbon microseepages in the Patrick Draw area using remote sensing techniques, much room exists for the continued development and application of these methodologies. For example, due to the lack of outcrop in the study area most analyses are based on soil 
samples. Because soils represent the weathered potion of the surrounding rock, their mineralogical signatures tend not to be as strong, both spectrally and geochemically. This situation is not always ideal for the Hyperion hyperspectral remote sensing system, which tends to be somewhat noisy and has a low spatial resolution of 30 by 30 meters. Thus, further analyses in the area might consider implementing a remote sensing system with a less noisy spectral resolution and higher spatial resolution so as to help characterize even more subtle alterations associated with hydrocarbon microseepages that can become lost in the already weathered soil conditions.

\section{METHODOLOGY}

Methods used in this study included field work, hyperspectral data analysis and geochemical laboratory work in an attempt to establish relationships between hyperspectral data and microseeping hydrocarbons within the Patrick Draw oilfield of south-central Wyoming (Figure 1). The following sections describe in further detail each of the steps taken to achieve this objective.

\subsection{Field Work}

Field work took place over the summer of 2005, during which the following objectives were accomplished:

1. Located surface lineaments along which the presence of microseeping hydrocarbons was confirmed in previous literature.

2. Collected soil samples along transects crossing these lineaments so as to obtain samples both within and outside of potential areas of alteration due to microseeping hydrocarbons.

3. Located previously defined areas of stunted sage and collected soil samples within them.

4. Obtained field spectroscopy of soil, rock and vegetation within predefined areas of hydrocarbon microseepage to aid in later classification of the Hyperion image Lineament and stunted sage locations were based on maps from earlier literature, which had previously confirmed these locations as representing areas of hydrocarbon microseepages, through the use of soil gas surveys (Abrams et al. 1984). These maps were then scanned and georeferenced using corner coordinates, allowing for the exact latitude and longitude measurements to be obtained for all features. Local road maps of the area were then overlain with lineaments and areas of stunted sage for further reference (Figure 2). Through the use of a hand-held Trimble GeoXT Global Positioning System (GPS), as well as the georeferenced maps below, mapped lineament locations were successfully identified in the field (Figure 2).

\subsection{Remote Sensing Data Analysis}

The primary objective of the remote sensing data analysis was the successful classification of the Hyperion hyperspectral image as well as the identification of altered surfaces within this classification that represent the presence of microseeping hydrocarbons.

In contrast to previous studies in the Patrick Draw area, which used multispectral data, 
this study employed the use of hyperspectral data. Multispectral and hyperspectral remote sensing systems differ primarily in bandwidths. Bandwidths are defined as the width of the electromagnetic spectrum that a single channel of the sensor is receptive to.

Multispectral sensors have a smaller number of bands with larger bandwidths, which can cause diagnostic absorption and reflection characteristics over narrow wavelength intervals to become lost. However, hyperspectral sensors acquire images in many, very narrow, contiguous spectral bands, which allows for a higher spectral resolution and easier identification of materials compared to the broader band multispectral sensors that cannot resolve these diagnostic spectral differences (Lillesand, 2000).

A special request was made to USGS for Hyperion data acquisition, as a result a single scene covering Patrick draw area was acquired on July 22nd, 2005. The Hyperion product consists of a total of 242 spectral bands covering a complete spectrum from 357 $2576 \mathrm{~nm}$ at a spatial resolution of 30 meters. Because of an overlap between the VNIR and SWIR focal planes there are only 196 unique channels. Calibrated channels are 8-57 for the VNIR and 77-224 from the SWIR. The reason for not calibrating all 242 channels is mainly due to the detectors low responsivity or decreased sensitivity. The bands that are not calibrated are set to zero in those channels (Beck, 2003).

\subsubsection{Hyperion image processing}

The software used for all Hyperion data processing was ENVI 4.2 (the Environment for Visualizing Images). The original Hierarchical Data Format (HDF file format was converted to ENVI standard format. There are 46 bands within the Hyperion dataset that are uncalibrated. These bands are set to zero by the collection agency and are considered useless and were removed before applying any further processing. The removal of these bands leaves only 196 bands for further processing. Using ENVI's FLAASH method, or Fast Line-of-Sight Atmospheric Analysis of Spectral Hypercubes the data was corrected for atmospheric influences.

\subsubsection{Image Classification}

Classification is the process of assigning pixels in an image to different classes based on their spectral character. These different classes can then be mapped as land cover type. There are two primary types of classifications, supervised and unsupervised classification. Supervised classification uses spectral characteristics of specific sites identified within the image or spectral characteristics of endmembers input by the user to classify the rest of the scene for spectral mapping. Unsupervised classification merely groups pixels with similar spectral characteristics within the image into clusters with no further inputs. This project employed ENVI's Spectral Mapping Wizard supervised classification. The Spectral Mapping Wizard is a guide that walks the user through the different steps required for supervised image classification.

\subsection{Geochemistry}

2.3.1 Laboratory Spectroscopy

Using the ASD Spectroradiometer in Khan's GeoRS laboratory, laboratory spectroscopy analysis was performed on all the samples collected in the field. The objective of this analysis was two twofold. The first objective is to aid in the classification of the Hyperion 
image. The second objective is the use of spectra taken in the laboratory for mineral identification of samples collected from the field.

Mineralogy of samples can be readily identified based on reflectance patterns of laboratory spectroscopy. This data was also used to compliment XRD analyses in an attempt to identify mineral compositions of samples to be collected in the field based on sample reflectance patterns. This will be done in order to help establish relationships between mineralogical alterations caused by microseeping hydrocarbons and to identify mineralogy of collected samples.

\subsubsection{XRD Analysis}

Powder X Ray Diffraction, or XRD, analysis was performed on the soil and sediment sample from Patrick Draw area using UH's Siemen D5000 X-ray Diffractometer for mineral identification. The goal of this analysis was to potentially identify mineralogical compositions of samples and to distinguish whether these compositions could be related to hydrocarbon microseepage.

\subsubsection{ICP Analysis}

To be able to identify elemental concentrations, inductively coupled plasma atomic emission spectroscopy (ICP-AES) analysis was performed on 32 samples collected from the field. The goal of this work was to aid in geochemical mapping of potential areas of alteration related to hydrocarbon microseepage.

\subsubsection{Carbon Isotope Analysis}

Selected samples collected from the field (Figure 2) were analyzed for carbon isotope ratios in an attempt to confirm the hydrocarbon source for surface alterations as being the underlying reservoir. Sample locations were chosen for analysis based on their increased reactivity to hydrochloric acid. We used UH's stable isotope facility which is equipped with two computer-controlled stable isotope ratio mass spectrometers (Finnegan MAT Delta $\mathrm{E}$ and Micromass 602) capable of measuring the isotope ratios ${ }^{2} \mathrm{H} /{ }^{1} \mathrm{H},{ }^{13} \mathrm{C} /{ }^{12} \mathrm{C}$, ${ }^{15} \mathrm{~N} /{ }^{14} \mathrm{~N},{ }^{18} \mathrm{O} /{ }^{16} \mathrm{O}$, and ${ }^{34} \mathrm{~S} /{ }^{32} \mathrm{~S}$.

\section{RESULTS AND INTERPRETATION}

\subsection{Remote Sensing Results and Interpretation}

Classification outputs from the Spectral Hourglass Wizard described earlier included Spectral Angle Mapper (SAM) results as well as Mixture Tuned Matched Filter (MTMF) results. Spectral Angle Mapper is a method for directly comparing image spectra to known spectral endmembers input by the user and/or defined from the image spectra itself. For this project, user input endmembers consisted of three different types of spectra: 1) spectra collected from the field, 2) spectra collected in the laboratory of analogous samples collected in the field, and 3) spectra downloaded from a spectral library based on mineralogies identified within the same samples collected from the field. Endmembers were also derived from the image itself, which is achieved through an automated process of ENVI software. This process defines endmembers based on different spectral extremes identified from pixel spectra within the image. The SAM method then treats unknown image spectra and endmember spectra (based on user 
endmember input or image endmember definition) as vectors and calculates the spectral angle between them (Research Systems, 2001a). The resulting SAM classification of an image identifies different color-coded classes based on the best match between image and endmember spectra at each pixel. This image can then be used to identify different materials at the surface.

MTMF is a classification method that also provides a means of detecting specific materials based on matches to user endmember input or image-derived endmember spectra. However, unlike SAM, which picks the best match to a given spectrum in the image, MTMF first maximizes the response of the known endmembers and suppresses the response of the unknown background signal in the image prior to matching. MTMF also utilizes a mixing model to determine what materials cause a particular spectral signature in image spectral data, as pixels are rarely composed of a single material. SAM analysis proved to be more effective in the classification of anomalous areas, or areas that are distinct from their surroundings.

When using the Spectral Hourglass Wizard several different user inputs for endemembers can be entered for classification. For this project, images were classified using four different types of spectral endmembers:

1. Spectra taken in the field using an ASD Spectroradiometer over previously defined areas of hydrocarbon microseepage.

2. Spectra taken in the laboratory also using an ASD Spectroradiometer on samples collected from the field covering the same areas.

3. Spectra collected from the USGS spectral library of minerals that were identified in samples based on spectral analysis and XRD results.

4. Spectra identified within the image based on spectral extremes identified from pixel spectra.

Each of these endmembers (except for those derived from the image itself), represents spectra collected over areas of predefined hydrocarbon microseepage. Thus, their identification as classes that are distinct from other classes within the image provides evidence for their definition as areas with spectral signatures associated with alterations due to hydrocarbon microseepage. Also, in the following sections, geochemical data is used in an attempt to demonstrate the association of areas defined by these classes with hydrocarbon microseepages. The resulting images of these four different types of classification are shown in Figure 3. The images were classified using spectra taken from the field (type 1), lab (type 2), and spectral library (type 3) as endmembers, and also included classes based on spectral endmembers derived from image itself (type 4). The ENVI software allows the user to see exactly what endmembers were used to define what class, and it was found that only classes defined using laboratory spectra (type 2) and image endmembers (type 4) were identifiable within the image. In other words, spectral endmembers based on field spectra (type 1) and spectral library data (type 3 ) of areas of hydrocarbon microseepage were not identified within the image. The reasoning as to why field and library spectral endmembers were not classified within the scene could be due to a number of factors. Most likely spectra of specific minerals based on spectral library inputs were not identified due to the low spatial resolution of the image, which is 30 by 
30 meters. Low spatial resolution causes the larger pixels to be a mixture of mineral assemblages rather than single minerals, making the classification of single mineral spectra more difficult. However, the reasons as to why field spectra were not identified within the image is not as straightforward, since one would expect spectroscopy taken in the field to be the most similar to spectroscopy taken by a satellite. One potential explanation could be differing environmental influences due to the fact that the field and satellite spectra were not measured on the exact same day. Another explanation could be that there was noise introduced to the field spectra by the machinery used to measure reflectance properties in the field, causing the satellite and field spectra to differ from one another.

Not all spectra taken in the laboratory of samples collected from the field were identified in the SAM classification of the scene. However, the ones that were included in the classification defined different zones. These zones were defined by looking at the spatial distribution of the identified classes illustrated in Figure 3 separately. Most zones merely correlate with known local surface geology. However, one zone, when looked at separately from other classes, defines a northeast to southwest trend through the field not previously identified within the local geology (Figure 4A). Because this zone is not identified within the local geology it is considered anomalous. Correlations between this zone and previously identified areas of hydrocarbon microseepage are shown in Figure 4B. This anomalous zone occasionally correlates with previously identified sample sites of hydrocarbon microseepage and their associated lineaments, but the more prominent pattern is a broad NE to SW trend rather than several, more local east/west trends following lineament patterns. Similarly, there is no relationship between this trend and previously mapped subsurface faults in the area (Figure 4C). However, upon further investigation of the local geology, a transitional member between two stratigraphic units, the Fort Union and the Wasatch, was noted to have a trend similar to that of the anomalous zone. This transitional member consists of a prominent, resistant, 5 meter thick carbonate cemented sandstone (Abrams, 1984). To confirm the correlation between trends, this transitional member, based on locations from geological maps of the area (Abrams, 1984), was overlain with the hyperspectral data (Figure 4D). Figure 4D demonstrates that this anomalous zone or class identified from the hyperspectral data based on spectral input from zones of microseping hydrocarbons, does not follow the lineaments identified on the surface, but rather approximately delineates the transitional member between the Wasatch and Fort Union stratigraphic units.

\subsection{Geochemistry Results and Interpretation}

3.2.1 Lab Spectroscopy Results and Interpretation

Mineral compositions of anomalous samples identified within the Hyperion scene were compared only with those samples collected along the same lineament due to the fact that samples collected outside of this area are believed to represent different formations and potentially different weathering conditions. Therefore, comparisons between anomalous samples and samples collected within these different areas might only represent differences in formation makeup and/or weathering rates rather than geochemical alterations due to microseeping hydrocarbons within the same formation. 
Figure 5 illustrates the different mineralogical compositions between anomalous samples and the remaining samples collected from within the same area. One noticeable trend is the predominance of clay minerals within the anomalous samples compared to the nonanomalous samples, except for Illite, which is more prevalent in the non-anomalous samples. This trend can be explained by the fact that the production of $\mathrm{CO}_{2}, \mathrm{H}_{2} \mathrm{~S}$, and organic acids resulting from the microbial oxidation of hydrocarbons in near-surface soils and sediments can create reducing, slightly acidic conditions that promote the diagenetic weathering of feldspars to produce clays and may lead to the conversion of normally stable illitic clays to kaolinite (Schumacher, 1996).

\subsubsection{XRD Results and Interpretation}

Like laboratory spectroscopy analyses, XRD results were focused on mineralogical comparisons between samples defining the anomalous zone within the classified Hyperion scene and samples collected within the same area. Once again this was done so as to avoid the influence of sampling pattern effects. Unlike laboratory spectroscopy analyses, which were more sensitive to clay mineralogies, XRD analysis revealed feldspar compositions. The lack of clay mineralogies in the XRD results could be due to a the intensity of the silica peak at approximately $25^{\circ}$ which is very high and all other peaks with lower intensities, such as those associated with clay mineralogies, are so dampened that they are undistinguishable from noise.

\subsubsection{ICP Results and Interpretation}

Anomalous samples consistently have lower values than non-anomalous samples for both major and minor elemental concentrations, except in the case of calcium (Figure 6). Bacterial oxidation of microseeping hydrocarbons not only results in the production of new minerals or the preferential accumulation of unaffected residual minerals, as demonstrated in the XRD and laboratory spectroscopy analyses, but it also results in the removal of soluble constituents from the weathering site (Schumacher, 1996). These soluble constituents include those elements listed in Figure 6, which explains why their occurrence would be diminished in the presence of an oxidizing environment associated microseeping hydrocarbons compared to their unaltered surroundings. However, the prevalence of $\mathrm{CaO}$ can be attributed to the fact that digenetic carbonates and carbonate cements are among the most common hydrocarbon-induced alterations associated with petroleum seepage (Schumacher, 1996). Once again, these carbonates are formed principally as a byproduct of hydrocarbon oxidation by microorganisms and whose predominance over other elemental abundances is reflected in ICP analyses.

\subsubsection{Carbon Isotope Results and Interpretation}

The carbon in normal calcite, whether derived from the atmosphere, freshwater or marine environment, has a carbon isotopic value of about -10 to $+5 \%$ relative to the PDB standard. The carbon isotopic composition of most crude oils ranges from approximately -20 to $-32 \%$, whereas that of methane can range from -30 to $-90 \%$. Calcite formed from oxidized petroleum, such as in the case of microseeping hydrocarbons, incorporates carbon from an organic source, which typically has an isotopic composition more 
negative than $-20 \%$ (Schumacher 1996). Isotopic compositions obtained from within the Patrick Draw study area recorded values as low as $-45.321 \%$ (Table 1 ).

\section{CONCLUSIONS}

The overall goal of this project was to build upon and implement a methodology for using remote sensing imagery to identify and map alteration zones caused by hydrocarbon microseepage in the Patrick Draw area of Southwest Wyoming. Previous attempts in the area have had to rely on spectral anomalies relating to stunted surface vegetation to identify underlying microseeps. This was because the broad bandwidth of the spectral imagery that was used was unable to characterize the more subtle absorption features caused by hydrocarbon microseepage induced alterations. However, the direct relationship between vegetation growth and microseepages has recently come in to question, giving more weight to the influence of injected gases and waters to maintain reservoir pressures rather than microseeping hydrocarbons (Arp, 1992).

Both remote sensing and geochemical techniques were applied to discern areas of hydrocarbon microseepage. Remote sensing methods employed the use of a number of different classification techniques in an attempt to identify anomalous areas located within the vicinity of previously defined areas of hydrocarbon microseepage. Training the image with endmemebrs based on laboratory spectroscopy taken from samples collected within these areas of hydrocarbon microseepage, resulted in the successful identification of an anomalous zone. It was also found that SAM classification techniques were more successful in identifying anomalous zones than other types of classifications. This anomalous zone consisted of a northeast to southwest trend crossing the field that followed a defined stratigraphic boundary, which presumably forms a migrational pathway for hydrocarbon seepage. The geochemistry of samples from the anomalous zone was compared with nearby samples collected outside the anomalous zone.

Geochemical techniques, including ICP, XRD, spectroscopy and carbon isotope analyses, were used to identify geochemical alterations associated with the oxidation of migrating light hydrocarbons at the surface. Spectroscopy results demonstrated higher proportions of clays within the anomalous samples compared to non-anomalous samples, along with greater amounts of quartz in non-anomalous samples relative to anomalous samples. XRD analyses demonstrated the increased presence of feldspars in non-anomalous samples compared to anomalous samples. ICP results provided evidence for the removal of soluble constituents from the weathering sites of anomalous samples versus nonanomalous samples. All of these analytical results are consistent with geochemical alterations defined for oxidating surface hydrocarbons, supporting the presence of hydrocarbon microseepages defined by anomalous sample areas. Carbon isotope analyses did result in the identification of potential areas of hydrocarbon microseepages, but samples defining these areas were not recognized within the Hyperion classification. However, locations of these samples did fall within the area defined by the anomalous samples, which still supports the presence of microseeping hydrocarbon within this zone.

Results from Hyperion hyperspectral remote sensing work at the Patrick Draw oil field of southwest Wyoming demonstrates a successful application of hyperspectral mapping techniques when applied to the identification of subtle geochemical alterations associated 
with hydrocarbon microseepage. Methods used in this research also help to provide a foundation upon which further hydrocarbon exploration techniques can grow.

\section{REFERENCES}

Abrams, M.J., Conel, J.E., Lang, H.R. and Paley, H.N., 1984. The Joint NASA/Geostat Test Case Project: Final Report. AAPG, part 2, v.2, p. 11-1 to 11-98.

Arp, G.K., 1992. An Integrated Interpretation for the Origin of the Patrick Draw Oil Field Sage Anomaly. AAPG, v. 76, no. 3 p. 301-306.

Beck, R., 2003. EO-1 Users Guide. Cincinnati, OH, University of Cincinnati. p. 4-74

Lillesand, T.M., and Kiefer, R.W., 2000. Remote Sensing and Image Interpretation. New York, John Wiley and Sons Inc. p.1-52, 309-605.

Research Systems, 2001a. ENVI User's Guide. Boulder, CO, Research Systems, Inc.

Schumacher, D., 1996. Hydrocarbon-Induced Alteration of Soils and Sediments. in D. Schumacher and M.A. Abrams, eds., Hydrocarbon Migration and Its NearSurface Expression. AAPG, Mem. 66, p. 71-89. 
Table 1: Carbon isotopic compositions based on comparison to PDB standard

\begin{tabular}{|c|c|}
\hline Sample Number & $\delta^{13} \mathrm{C}$ \\
\hline $1 D$ & -5.331 \\
\hline $3 A$ & -2.881 \\
\hline $3 D$ & -4.484 \\
\hline $5 E$ & -45.321 \\
\hline $6 A$ & -9.466 \\
\hline $6 B$ & -34.523 \\
\hline
\end{tabular}




\section{FIGURES}

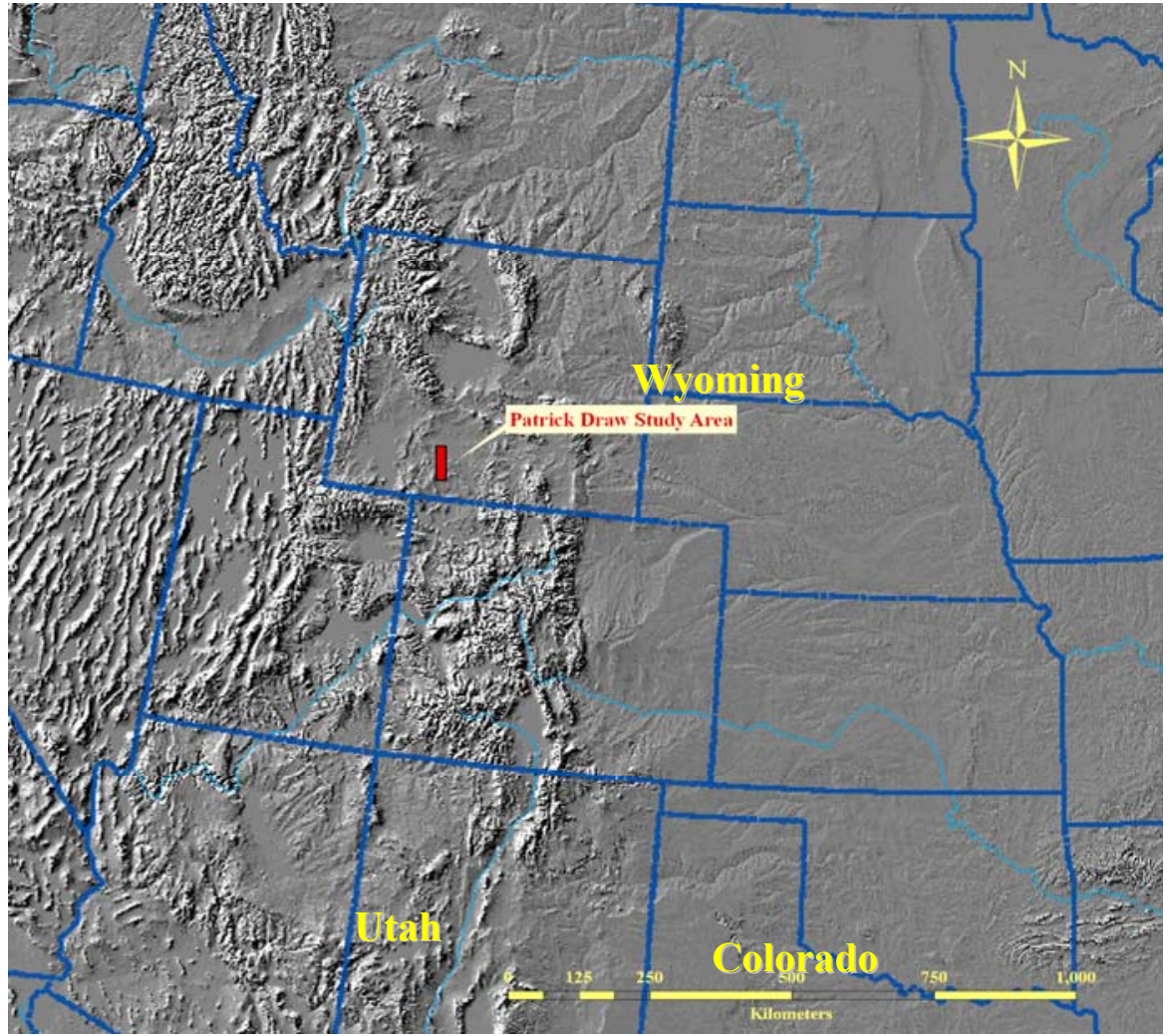

Figure 1: Shaded elevation map showing the location of the Patrick Draw study area within the foreland of the Rocky Mountain region. 


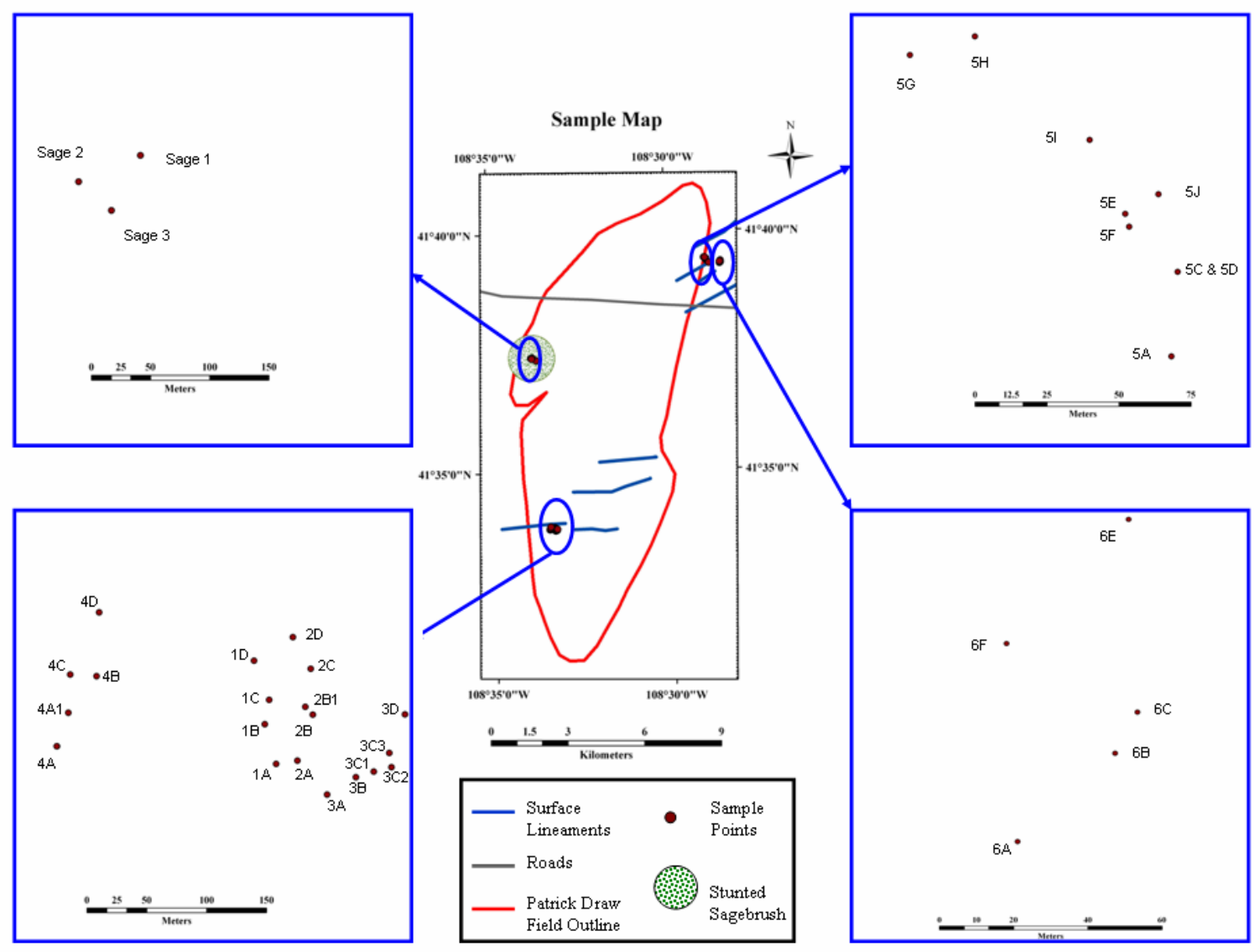

Figure 2: Map showing locations of all samples collected in the field with their labeled names 
(A)

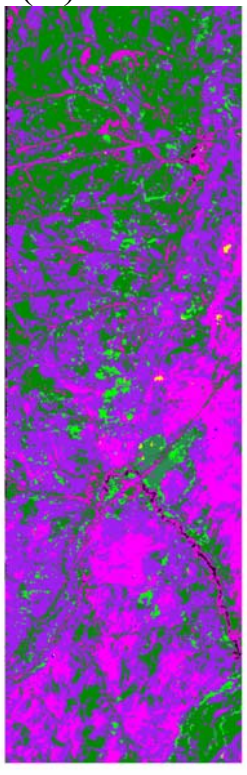

Classification Based on Field Spectroscopy

(C)

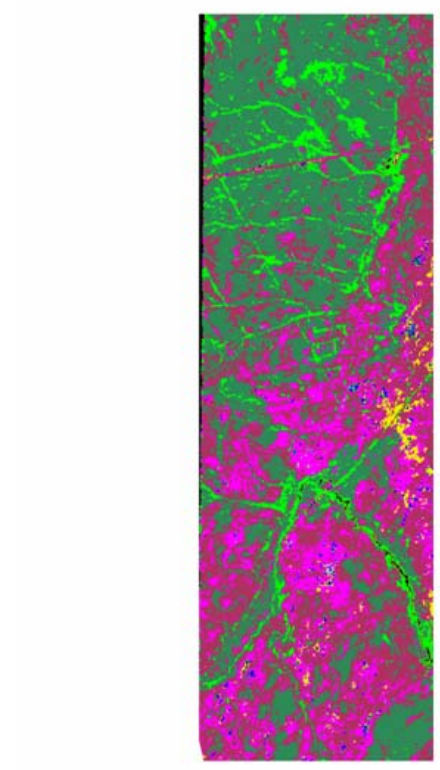

Classification Based on Library Spectroscopy
(B)

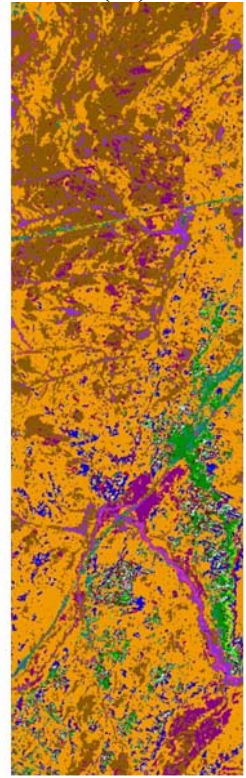

Classification Based on Lab Spectroscopy

(D)

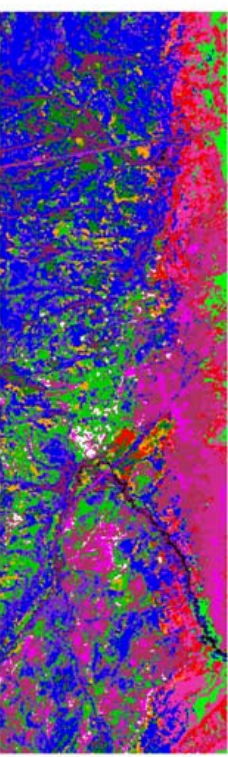

Classification Based on Image Spectroscopy
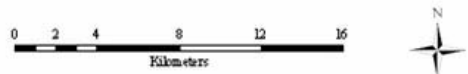

Figure 3: Classification results covering Patrick Draw area (A) Classification based on field spectroscopy (B) Classification based on laboratory sepectroscopy (C) Classification based on Library Spectrosocpy (D) Classification based on Image Spectroscopy 
(A)
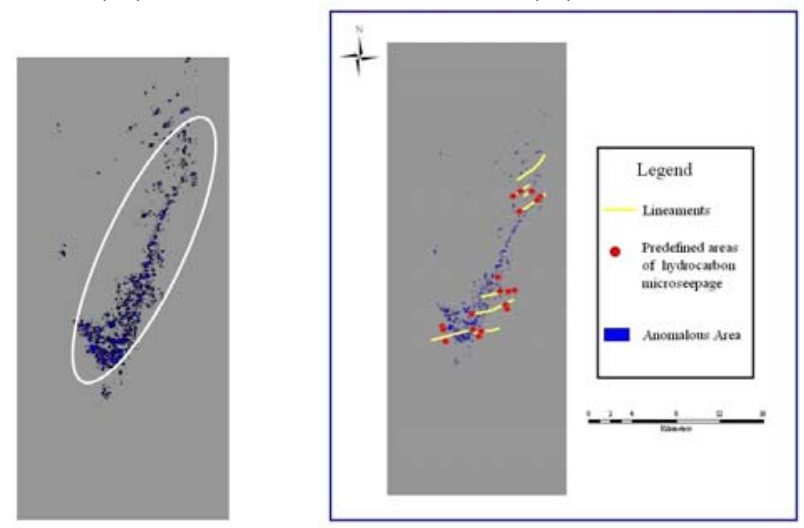

(D)

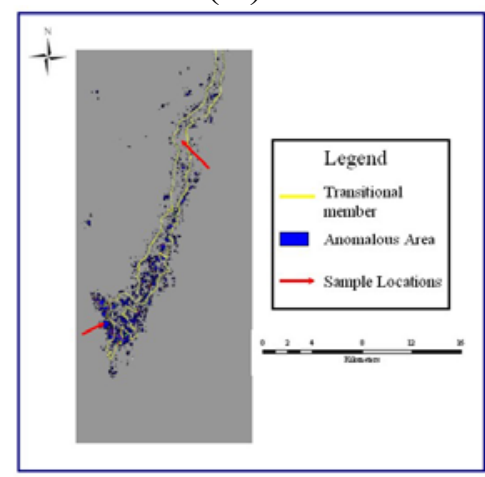

(C)

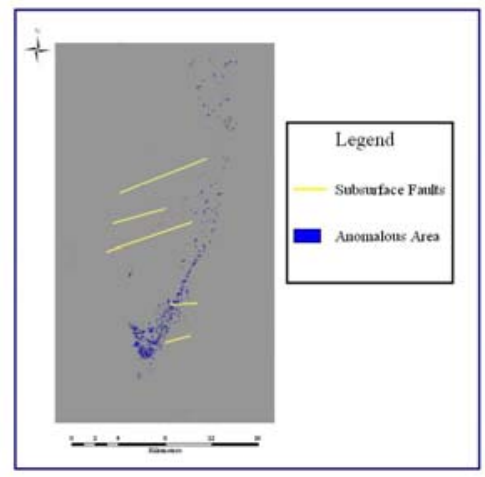

(E)

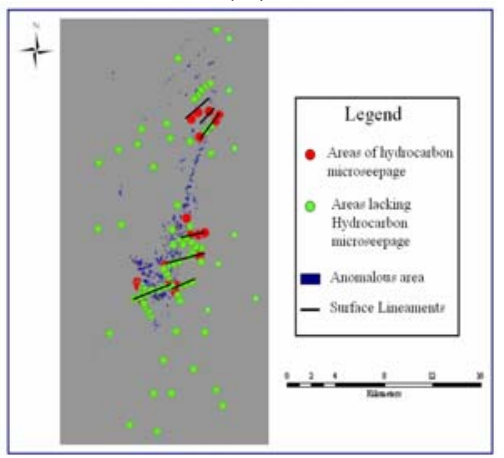

Figure 4: (A) Anomalous class in blue extracted (B) Anomalous area overlain with predefined areas of hydrocarbon microseepage and their associated lineaments in Patrick Draw study area (C) Overlay of mapped subsurface faults with anomalous zone in Patrick Draw (D) Overlay of transitional member (Abrams, 1984) with anomalous area at Patrick Draw; red arrow shows locations for carbon isotope samples. (E) Spatial trend of previously identified areas of hydrocarbon microseepage 


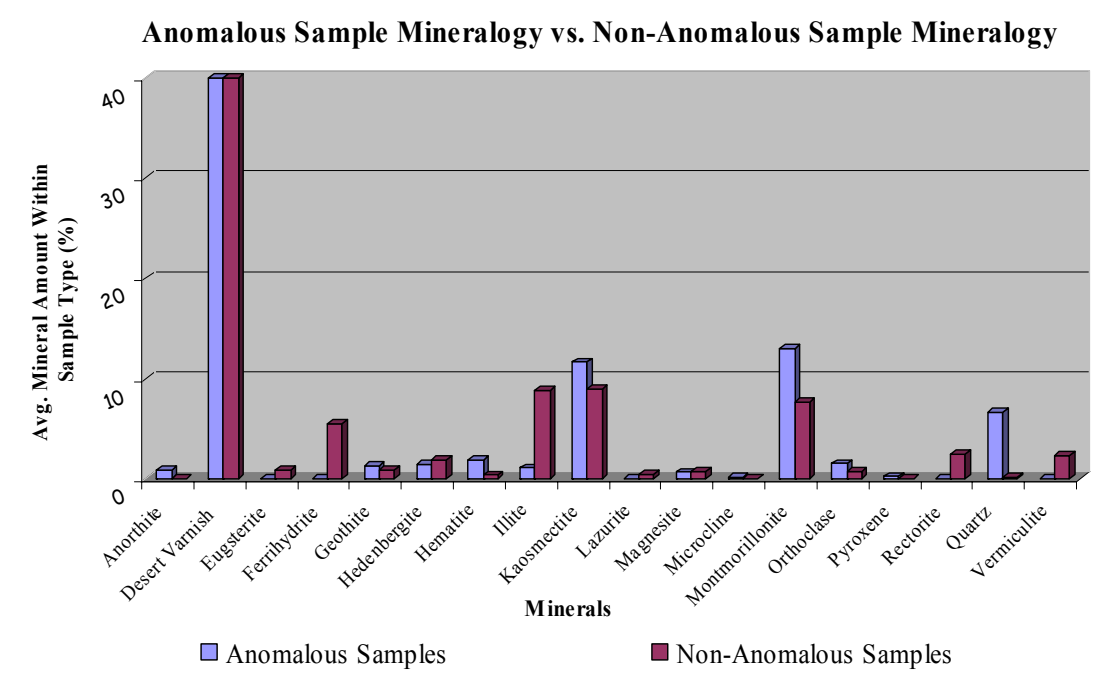

Figure 5: Bar graph showing mineralogical composition based on laboratory spectroscopy

Major Elemental Abundances of Anomalous Samples vs. Non-Anomalous Samples

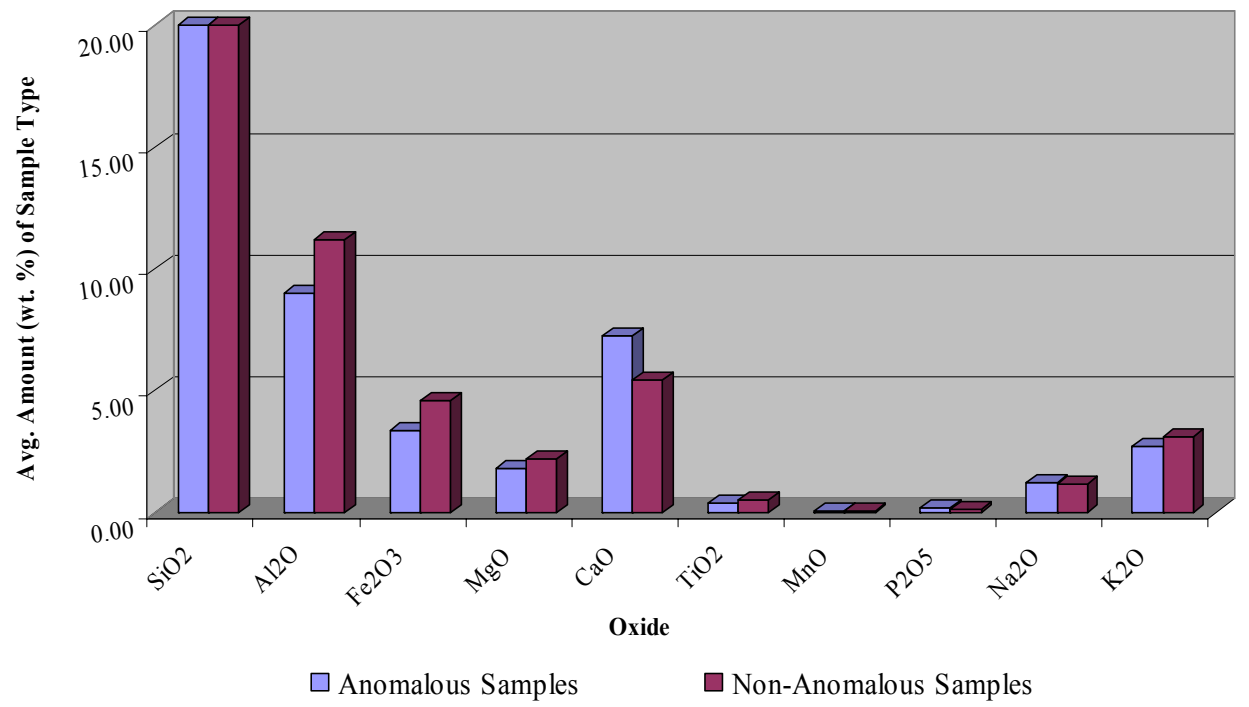

Figure 6: (A) Major elemental abundances based on ICP analyses (B) Minor elemental abundances based on ICP analyses 